\title{
Bowel Obstruction Management
}

National Cancer Institute

\section{Source}

National Cancer Institute. Bowel Obstruction Management. NCI Thesaurus. Code C51995.

Techniques and procedures for maintaining the health of patients with obstructive bowel disease, 